Georgian Mathematical Journal

Volume 14 (2007), Number 2, 351-360

\title{
MULTIPLICITY OF SOLUTIONS FOR SECOND ORDER TWO-POINT BOUNDARY VALUE PROBLEMS WITH ASYMPTOTICALLY ASYMMETRIC NONLINEARITIES AT RESONANCE
}

\author{
FELIX SADYRBAEV
}

\begin{abstract}
Estimations of the number of solutions are given for various resonant cases of the boundary value problem $x^{\prime \prime}+g(t, x)=f\left(t, x, x^{\prime}\right)$, $x(a) \cos \alpha-x^{\prime}(a) \sin \alpha=0, x(b) \cos \beta-x^{\prime}(b) \sin \beta=0$, where $g(t, x)$ is an asymptotically linear nonlinearity, and $f$ is a sublinear one. We assume that there exists at least one solution to the BVP.
\end{abstract}

2000 Mathematics Subject Classification: 34B15.

Key words and phrases: Nonlinear boundary value problems, jumping nonlinearities, multiplicity of solutions, resonance, angular function.

\section{INTRODUCTION}

Theory of nonlinear boundary value problems for ordinary differential equations is a rapidly growing field of applied analysis. There are more than twenty monographs devoted to the subject and a lot of books containing several chapters on nonlinear BVPs. We mention those by Bernfeld and Lakshmikantham [1], Kiguradze [11] and Vasilyev and Klokov [12]. Of the most recent ones there is a book by De Coster and Habets [2].

A second order nonlinear equation together with two-point boundary conditions still remains a popular object for investigations. Numerous methods of topological, variational, functional-analytic, computational nature are used to solve the basic problems of the theory. These are the existence of solutions (also the nonexistence), properties of solutions and estimations of the number of solutions. In treating these problems, researchers often try to reduce the original problem to a quasi-linear one, which has a linear part and a nonlinear one which is bounded (or sublinear). For instance, the Dirichlet boundary value problem $x^{\prime \prime}=f\left(t, x, x^{\prime}\right), x(a)=A, x(b)=B$ can be reduced (in some cases) to the quasi-linear problem $x^{\prime \prime}=F\left(t, x, x^{\prime}\right), x(a)=A, x(b)=B$, where $F$ is a bounded nonlinearity. The solvability of the latter problem follows from the well-known results if the related homogeneous problem $x^{\prime \prime}=0, x(a)=0$, $x(b)=0$ is non-resonant, that is, it has the trivial solution only. The reduced quasi-linear equation may also have the form $x^{\prime \prime}+p(t) x^{\prime}+q(t) x=F\left(t, x, x^{\prime}\right)$ with a richer linear part. The non-resonance property of the linear part in a functional analytic interpretation means that a respective linear operator is invertible, that is, it has a zero kernel. 
Additional difficulties appear if the problem is under resonance in the sense that the corresponding homogeneous problem has nontrivial solutions, too. An example is the Dirichlet problem for the equation $x^{\prime \prime}+x=F\left(t, x, x^{\prime}\right)$, to which the corresponding homogeneous one $x^{\prime \prime}+x=0, x(0)=0, x(\pi)=0$ has a continuum of nontrivial solutions. Nevertheless, for the specific right sides $F$ the nonlinear problem may have exactly a prescribed number of solutions.

A step towards considering more complicated problems was done in [3], where the piece-wise linear part $x^{\prime \prime}+a x^{+}-b x^{-}$was discussed $\left(x^{+}=\max \{x, 0\}\right.$, $\left.x^{-}=\max \{-x, 0\}\right)$, which in some sense is similar to the linear one. The Fučik equation $x^{\prime \prime}+a x^{+}-b x^{-}=0$ is a limiting case of the nonlinear equation

$$
x^{\prime \prime}+g(x)=F\left(t, x, x^{\prime}\right),
$$

where $g(x)$ behaves like the linear function $a x($ resp. bx) at $+\infty$ (resp. at $-\infty)$ and the right side $F$ is bounded. Such equations are now called equations with asymptotically asymmetric nonlinearities. Since the left side in (1) is "almost linear", its resonant or non-resonant behavior heavily affects the solvability of the related boundary value problems.

In what follows we consider the resonant cases of the boundary value problem

$$
\begin{gathered}
x^{\prime \prime}+g(t, x)=f\left(t, x, x^{\prime}\right), \\
x(a) \cos \alpha-x^{\prime}(a) \sin \alpha=0, \quad \alpha \in[0, \pi), \\
x(b) \cos \beta-x^{\prime}(b) \sin \beta=0, \quad \beta \in(0, \pi],
\end{gathered}
$$

where the nonlinearity $g(t, x)$ is asymptotically linear and, possibly, asymmetric, that is, the limits $g_{+}=\lim _{x \rightarrow+\infty} \frac{g(t, x)}{x}$ and $g_{-}=\lim _{x \rightarrow-\infty} \frac{g(t, x)}{x}$ exist and may be distinct.

Problems of this kind were studied intensively under the assumption that $g_{-}<g_{+}$(the case of $g_{+}<g_{-}$can be reduced to the above case by changing the variable $x$ to $-x$ ). The interested reader may trace the stages of investigation of boundary value problems with "jumping nonlinearities" (or, in alternative terminology, asymptotically asymmetric equations) following the works [15], [20], [6], [21], [17] and the references therein.

A natural way to consider a problem like (2), (3) is to compare it with the associated linear eigenvalue problem

$$
\begin{gathered}
x^{\prime \prime}+\lambda x=0, \\
x(a) \cos \alpha-x^{\prime}(a) \sin \alpha=0, \\
x(b) \cos \beta-x^{\prime}(b) \sin \beta=0,
\end{gathered}
$$

which, by classical results, has a countable set of eigenvalues $\left\{\lambda_{1}, \lambda_{2}, \ldots\right\}$ of multiplicity 1.

The cases of $\left(g_{-}, g_{+}\right) \subset\left(-\infty, \lambda_{1}\right)$ and $\left(g_{-}, g_{+}\right) \subset\left(\lambda_{k}, \lambda_{k+1}\right)$ can be characterized as "good" ones, when considering the solvability of problems with general right sides $f$.

Problems with a nonlinearity $g$ "crossing" several eigenvalues, that is

$$
g_{-}<\lambda_{1}<\ldots<\lambda_{k}<g_{+}<\lambda_{k+1} \quad \text { or }
$$




$$
\lambda_{n}<g_{-}<\lambda_{n+1}<\ldots<\lambda_{n+k}<g_{+}<\lambda_{n+k+1}
$$

seem to be more complicated. Problems of this type are very likely to have multiple solutions for appropriate right sides $f$. The estimations of the number of solutions were obtained, for example, in the works [3], [5], [6], [7], [20]. Mostly, the cases of constant limits $g_{+}$and $g_{-}$and $f$ depending on $t$ and some parameters only were considered.

It was observed that a lower bound for the number of solutions to the problem (2), (3) generally increases along with the number of eigenvalues being crossed by a nonlinearity $g$ though for the specific right sides $f$ only. It is an easy matter to construct examples of problems of the type (2), (3) with $g$ crossing arbitrarily many eigenvalues and having at most one solution. The problem $x^{\prime \prime}+c^{2} x^{+}=0, x(0)=0=x(\pi)$, for instance, has only a trivial solution for any $c>1$ (here $g_{-}=0, g_{+}=c^{2}$ ).

This paper uses the technical background and ideas of [21], where nonresonant boundary value problems were considered. It was observed in [21] that usual assumptions on $g$, crossing eigenvalues of the linear problem (4), (5), and on the right side $f$ imply the existence of a particular solution $\xi(t)$ of the problem (2), (3). The properties of this particular solution, expressed in terms of oscillatory behavior of the corresponding linear equation of variations

$$
y^{\prime \prime}+g_{x}(t, \xi(t)) y=f_{x}\left(t, \xi(t), \xi^{\prime}(t)\right) y+f_{x^{\prime}}\left(t, \xi(t), \xi^{\prime}(t)\right) y^{\prime},
$$

where $g_{x}, f_{x}$ and $f_{x^{\prime}}$ stand for the respective partial derivatives, heavily affect the number of solutions to the BVP. It appears that the estimation from below of the number of solutions to the problem (2), (3) depends also on the properties of the asymptotically linear equation

$$
z^{\prime \prime}+g_{+}(t) z^{+}-g_{-}(t) z^{-}=0,
$$

where $z^{+}(t)=\max (z(t), 0), z^{-}(t)=z^{+}-z(t)$. This equation describes the behaviour of solutions of the nonlinear equation (2) at infinity (that is, the behaviour of solutions with large initial values $\left.x^{2}(a)+x^{2}(a)\right)$.

We use the angular function technique described in the book [13], Ch. 15. This technique for asymptotically asymmetric nonlinearities was used in [21] and, within the functional analytic framework instead of shooting one, was used for the study of other questions related to boundary value problems with jumping nonlinearities in [10].

Our assumptions on $g$ and $f$ are:

(A1) $g, f, g_{x}(t, x), f_{x}\left(t, x, x^{\prime}\right), f_{x^{\prime}}\left(t, x, x^{\prime}\right)$ are continuous;

(A2) the limits $g_{+}(t)=\lim _{x \rightarrow+\infty} \frac{g(t, x)}{x}$ and $g_{-}(t)=\lim _{x \rightarrow-\infty} \frac{g(t, x)}{x}$ exist and are uniform in $t \in[a, b]$;

(A3) $f(t, x, y)$ is sublinear, that is $\frac{f(t, x, y)}{|x|+|y|}$ tends to zero as $|x|+|y|$ tends to infinity, uniformly in $t \in[a, b]$;

(A4) there exists a solution $\xi$ to the problem (2), (3). 
Obviously, solutions of the equation (2) exist on the interval $[a, b]$, are uniquely determined by the initial conditions and depend continuously on the initial data and on the functions $g(t, x)$ and $f(t, x, y)$.

\section{Preliminary Results}

In this section, we provide some necessary tools for our investigation. Let $\xi(t)$ be a particular solution to the problem (2), (3).

Introduce the polar coordinates by

$$
u:=x-\xi=\rho \sin \phi, \quad u^{\prime}=x^{\prime}-\xi^{\prime}=\rho \cos \phi .
$$

In our notation, the increase of $\phi(t)$ corresponds to the clock-wise rotation of a solution on a phase plane $\left(u, u^{\prime}\right)$.

Denote by $\phi_{+}(t, \rho)$ and $\phi_{-}(t, \rho)$ the angular functions defined by the conditions

$$
\begin{gathered}
\phi_{+}(a, \rho)=\alpha, \quad u^{2}(a)+u^{\prime 2}(a)=\rho^{2}, \\
\phi_{-}(a, \rho)=\alpha-\pi, \quad u^{2}(a)+u^{\prime 2}(a)=\rho^{2}
\end{gathered}
$$

respectively.

The angular functions for solutions of equations (6) and (7) can be introduced similarly. Notice that both the linear equation (6) and the piece-wise linear equation (7) possess the property of positive homogeneity. This means that if $w(t)$ is a solution to (6) or (7), then $c^{2} w(t)$ also is a solution, where $c$ is a constant. For positively homogeneous equations the angular function is defined only by the initial value of a polar angle and is independent of $\rho$.

Lemma 2.1. Let $\theta(t)$ be the angular function of solutions of the equation of variations $(6)$, defined by $\theta(0)=\alpha$.

Then $\phi_{+}(t ; \rho) \rightarrow \theta(t)$ as $\rho \rightarrow 0$, and $\phi_{-}(t ; \rho) \rightarrow \theta(t)-\pi$ as $\rho \rightarrow 0$ uniformly in $t \in[a, b]$.

Proof is accomplished by a standard argument (see, for example, [13], Theorem 15.11).

Lemma 2.2. Let $\theta_{+}(t)$ and $\theta_{-}(t)$ be the angular functions for solutions of $(7)$, defined by the initial conditions $\theta_{+}(0)=\alpha$ and $\theta_{-}(0)=\alpha-\pi$, respectively.

Then

$$
\begin{aligned}
& \phi_{+}(t ; \rho) \rightarrow \theta_{+}(t) \text { as } \rho \rightarrow \infty, \text { uniformly in } t, \\
& \phi_{-}(t ; \rho) \rightarrow \theta_{-}(t) \text { as } \rho \rightarrow \infty, \text { uniformly in } t .
\end{aligned}
$$

Proof. Use Lemma 3.3 from [21].

In the paper [21], the results were obtained assuming that for the angular functions $\theta(t), \theta_{+}(t), \theta_{-}(t)$, described in the above two lemmas the following conditions hold:

$$
\theta(b) \neq \beta(\bmod \pi), \quad \theta_{+}(b) \neq \beta(\bmod \pi), \quad \theta_{-}(b) \neq \beta(\bmod \pi) .
$$

It is natural to call these conditions nonresonant. 
In this paper, we consider the situation, where equality (or equalities) may occur in (11). We call these cases resonant and propose the classification of types of resonance described in the next sections.

\section{Symmetric Resonance}

In this section we consider the equation

$$
x^{\prime \prime}+g(t, x)=f\left(t, x, x^{\prime}\right)
$$

where

$$
\lim _{x \rightarrow+\infty} \frac{g(t, x)}{x}=\lim _{x \rightarrow-\infty} \frac{g(t, x)}{x}=: g(t)
$$

and the limits are uniform in $t \in[a, b]$.

Definition 3.1. We say that the problem (2), (3) is under symmetric $k$ resonance if the linear equation

$$
x^{\prime \prime}+g(t) x=0
$$

has a nontrivial solution satisfying the homogeneous boundary conditions (5), and the angular functions $\theta_{ \pm}(t)$ of solutions of (14) defined by the initial conditions

$$
\theta_{+}(a)=\alpha, \quad \theta_{-}(a)=\alpha-\pi,
$$

are such that both functions $\theta_{ \pm}(t)$ take exactly $k$ values of the form $\beta-\pi i$ when $t \in[a, b]$ with $\theta_{ \pm}(t)=\beta(\bmod \pi)$ (in fact $\left.\theta_{-}(t) \equiv \theta_{+}(t)-\pi\right)$.

Example. To illustrate the problem under symmetric 1-resonance consider

$$
x^{\prime \prime}+x+\omega(x)=0, \quad x(0)=0=x(\pi),
$$

where $\omega(x)=\frac{1}{x^{2}+1}$ for large values of $|x|$ and is smooth. Since solutions with $x(0)=0$ and $x^{\prime}(0)$ positive and large enough have a zero less than $\pi$, and solutions with $x(0)=0$ and $x^{\prime}(0)$ negative and large in modulus have a zero greater than $\pi$, there exists a solution $\xi(t)$ with $\xi(0)=0$ and $\xi(\pi)=0$. This solution may be trivial.

We introduce now

Definition 3.2. We say that a solution $\xi(t)$ of the problem (2), (3) is of index $n$ if the angular function $\theta(t)$ of a solution $y(t)$ of the equation of variations (6) defined by the initial data

$$
y(a) \cos \alpha-y^{\prime}(a) \sin \alpha=0, \quad y^{2}(a)+y^{\prime 2}(a)=1
$$

takes exactly $n$ values of the form $\beta+\pi i$ ( $i$ is an integer) in the interval $[a, b]$ and

$$
\theta(b) \neq \beta(\bmod \pi) .
$$

The following result provides the estimation of the number of solutions to the resonant problem (2), (3) under the assumption that there exists a specific solution $\xi(t)$. 
Theorem 3.1. Suppose the condition (A3) holds and the problem (2), (3) is under symmetric $k$-resonance. Let there exist a solution $\xi(t)$ of index $n$ of the problem (2), (3) and $n>k($ resp. $n<k)$.

Then there exist at least $2(n-k)+1$ (resp. $2(k-n-1)+1)$ solutions to the problem (2), (3), $\xi(t)$ counted.

Proof. Consider the angular functions $\phi_{+}(t, \rho)$ and $\phi_{-}(t, \rho)$ defined by $(9)$ and (10). Let the inequality $n>k$ hold. By lemma (2.1), the angular function $\phi_{+}(t, \rho)$ takes exactly $n$ values of the form $\beta+\pi i$ when $\rho$ is sufficiently small. On the other hand, $\phi_{+}(t, \rho)$ is close to $\beta+\pi k$ for $\rho$ large enough. Changing $\rho$ from zero to infinity, one gets at least $(n-k)$ solutions. Analogously, one can trace the behaviour of the function $\phi_{-}(t, \rho)$. Hence at least $2(n-k)+1$ solutions, $\xi(t)$ counted.

Consider the case with $n<k$. Changing $\rho$ from zero to infinity, one gets now at least $k-n-1$ solutions for both functions $\phi_{+}$and $\phi_{-}$.

\section{Asymmetric Resonance}

We consider now the problem (2), (3) under the assumption that

$$
g_{+}(t)=\lim _{x \rightarrow+\infty} \frac{g(t, x)}{x}>\lim _{x \rightarrow-\infty} \frac{g(t, x)}{x}=g_{-}(t)
$$

and the limits are uniform in $t \in[a, b]$.

Equation (7) is now substantial in our considerations and plays a role analogous to that played by equation (14) in the previous section.

The angular functions $\theta_{+}(t)$ and $\theta_{-}(t)$ defined in Lemma 2.2 can take now different numbers of values of the form $\beta(\bmod \pi)$ although these numbers may differ at most by unity (Proposition 2.1 in [21]).

We introduce the following definitions.

Definition 4.1. We say that the problem $(2),(3)$ is under $(+)$-asymmetric $(k, m)$-resonance if the angular functions $\theta_{+}(t)$ and $\theta_{-}(t)$ of the equation $(7)$ satisfy the conditions below:

1) $\theta_{+}(t)$ takes exactly $k$ values of the form $\beta(\bmod \pi)$ for $t \in[a, b]$ and $\theta_{+}(b)=\beta(\bmod \pi)$

2) $\theta_{-}(t)$ takes exactly $m$ values of the form $\beta(\bmod \pi)$ for $t \in[a, b]$ and $\theta_{-}(t) \neq \beta(\bmod \pi)$.

Definition 4.2. We say that the problem (2), (3) is under (-)-asymmetric $(k, m)$-resonance if the angular functions $\theta_{+}(t)$ and $\theta_{-}(t)$ of the equation $(7)$ satisfy the conditions below:

1) $\theta_{+}(t)$ takes exactly $k$ values of the form $\beta(\bmod \pi)$ for $t \in[a, b]$ and $\theta_{+}(b) \neq \beta(\bmod \pi)$

2) $\theta_{-}(t)$ takes exactly $m$ values of the form $\beta(\bmod \pi)$ for $t \in[a, b]$ and $\theta_{-}(t)=\beta(\bmod \pi)$.

Theorem 4.1. Suppose the condition (A3) holds and the problem (2), (3) is under either $(+)$-asymmetric or $(-)$-asymmetric $(k, m)$-resonance. Let there 
exist a solution $\xi(t)$ of index $n$ of the problem (2), (3) and $n>\max \{k, m\}$ (resp. $n<\min \{k, m\})$.

Then there exist at least $(n-k)+(n-m)+1$ (resp. $(k-n)+(m-n))$ solutions to the problem (2), (3), $\xi(t)$ counted.

Proof. Let the problem (2), (3) be under $(+)$-asymmetric $(k, m)$-resonance. Consider the angular functions $\phi_{+}(t, \rho)$ and $\phi_{-}(t, \rho)$ defined by (9) and (10). Let the inequality $n>\max \{k, m\}$ hold. By Lemma 2.1, the angular function $\phi_{+}(t, \rho)$ takes exactly $n$ values of the form $\beta+\pi i$ when $\rho$ is sufficiently small. On the other hand, $\phi_{+}(t, \rho)$ is close to $\beta+\pi k$ for $\rho$ large enough. Changing $\rho$ from zero to infinity, one gets at least $(n-k)$ solutions. Tracing the behaviour of the function $\phi_{-}(t, \rho)$ when $\rho$ changes from zero to infinity one gets at least $n-m$ solutions. Hence there are at least $(n-k)+(n-m)+1$ solutions, $\xi(t)$ counted.

Consider the case of $n<\min \{k, m\}$. Changing $\rho$ from zero to infinity, one gets now at least $k-n-1$ solutions for $\phi_{+}$and at least $m-n$ solutions for $\phi_{-}$. Hence at least $(k-n-1)+(m-n)+1=(k-n)+(m-n)$ solutions, $\xi(t)$ counted.

The case of the problem $(2),(3)$ being under $(-)$-asymmetric $(k, m)$-resonance can be treated analogously.

Example. Consider the problem $x^{\prime \prime}+g(x)=f(x), \quad x(0)=0=x(1)$, where $f$ and $g$ are $C^{1}$-functions such that $g(0)=f(0)=0, g_{x}(0)-f_{x}(0)=(7 \pi+\varepsilon)^{2}$ in some neighborhood of $x=0,0<\varepsilon<\pi$. This ensures that nontrivial solutions of the equation of variations with respect to $\xi \equiv 0$, which vanish at $t=0$, have exactly 7 zeros in the interval $(0,1)$ and do not vanish at $t=1$. Suppose also that $f$ is bounded and $g(x) \rightarrow(4 \pi)^{2} x$ as $x \rightarrow+\infty$ and $g(x) \rightarrow(2 \pi)^{2} x$ as $x \rightarrow-\infty$. The limiting equation (7) is now $z^{\prime \prime}+(4 \pi)^{2} z^{+}-(2 \pi)^{2} z^{-}=0$. It follows that $k=3, m=2$ and the boundary value problem has at least $(7-3)+(7-2)+1$ solutions, the trivial one counted.

\section{Double Resonance}

Definition 5.1. We say that the problem (2), (3) is under double asymmetric $(k, m)$-resonance if the angular functions $\theta_{+}(t)$ and $\theta_{-}(t)$ of the equation $(7)$ satisfy the conditions below:

1) $\theta_{+}(t)$ takes exactly $k$ values of the form $\beta(\bmod \pi)$ for $t \in[a, b]$ and $\theta_{+}(b)=\beta(\bmod \pi)$

2) $\theta_{-}(t)$ takes exactly $m$ values of the form $\beta(\bmod \pi)$ for $t \in[a, b]$ and $\theta_{-}(t)=\beta(\bmod \pi)$.

Theorem 5.1. Suppose the condition (A3) holds and the problem (2), (3) is under double asymmetric $(k, m)$-resonance. Let there exist a solution $\xi(t)$ of index $n$ of the problem (2), (3) and $n>\max \{k, m\}$ (resp. $n<\min \{k, m\})$.

Then there exist at least $(n-k)+(n-m)+1$ (resp. $(k-n)+(m-n)-1)$ solutions to the problem (2), (3), $\xi(t)$ counted.

Proof is analogous to the proof of Theorem 4.1. 
Remark. By double resonance we usually call (see, for example, [8]) the case of a boundary value problem of the type (2), (3), where $g_{+}=\lambda_{i}$ and $g_{-}=\lambda_{j}$ ( $\lambda$ 's are eigenvalues of the associated linear problem (4), (5)). However in this case the angular functions $\theta_{+}(t)$ and $\theta_{-}(t)$ need not satisfy the conditions

$$
\theta_{ \pm}(b)=\beta(\bmod \pi)
$$

and in fact the problem (2), (3) does not exhibit resonant behaviour.

Example. Consider the problem $x^{\prime \prime}+g(x)=f(x), \quad x(0)=0=x(1)$, where $f$ and $g$ are $C^{1}$-functions such that $g(0)=f(0)=0, g_{x}(0)-f_{x}(0)=(7 \pi+\varepsilon)^{2}$ in some neighborhood of $x=0,0<\varepsilon<\pi$. This ensures that nontrivial solutions of the equation of variations with respect to $\xi \equiv 0$, which vanish at $t=0$, have exactly 7 zeros in the interval $(0,1)$ and do not vanish at $t=1$. Suppose also that $f$ is bounded and $g(x) \rightarrow(6 \pi)^{2} x$ as $x \rightarrow+\infty$ and $g(x) \rightarrow(3 \pi)^{2} x$ as $x \rightarrow-\infty$. The limiting equation (7) is now $z^{\prime \prime}+(6 \pi)^{2} z^{+}-(3 \pi)^{2} z^{-}=0$. Notice that a solution $z^{*}(t)$ with $z(0)=0, z^{\prime}(0)=1$ has exactly 3 zeros in $(0,1)$ and vanishes at $t=1$. So does a solution $z_{*}(t)$ with $z(0)=0, z^{\prime}(0)=-1$, but they are not multiples of each other. This means that the problem is under double asymmetric $(4,4)$-resonance and the boundary value problem has at least $(7-4)+(7-4)+1$ solutions, the trivial one counted.

\section{INTERNAL RESONANCE}

Suppose that there exists a solution $\xi(t)$ of the problem (2), (3). Consider the equation of variations (6). Let $\theta(t)$ be the angular function of a solution of (6), defined by the initial conditions (15). It is natural to call the case of $\theta(t)=\beta(\bmod \pi)$ resonant.

Definition 6.1. We say that the problem (2), (3) is under internal $n$-resonance (with respect to $\xi$ ) if the angular function $\theta(t)$ of a solution $y(t)$ of the equation of variations (6) defined by the initial data (15) takes exactly $n$ values of the form $\beta+\pi i$ ( $i$ is an integer) in the interval $[a, b]$ and $\theta(b)=\beta(\bmod \pi)$.

Definition 6.2. We say that the problem (2), (3) is k-nonresonant if the angular functions $\theta_{ \pm}(t)$ of solutions of (14) take exactly $k$ values of the form $\beta+\pi i\left(i\right.$ is an integer) in the interval $[a, b]$ and $\theta_{ \pm}(b) \neq \beta(\bmod \pi)$.

Theorem 6.1. Suppose the condition (A3) holds and the problem (2), (3) is k-nonresonant. Suppose also that the problem (2), (3) is under internal $n$ resonance with respect to a particular solution $\xi$ of the $B V P$.

Then there exist at least $2(n-k)+1$ solutions to the problem $(2),(3), \xi(t)$ counted.

Example. Consider the problem $x^{\prime \prime}+g(x)=f(x), \quad x(0)=0=x(1)$, where $f$ and $g$ are $C^{1}$-functions such that $g(x)=(7 \pi)^{2} x$ in some neighborhood of $x=0, f(0)=f_{x}(0)=0$. This ensures that nontrivial solutions of the equation of variations with respect to $\xi \equiv 0$, which vanish at $t=0$, have exactly 7 zeros in the interval $(0,1]$ and vanish at $t=1$. Therefore the equation is under internal 7-resonance. Suppose also that $f$ is bounded and $g(x) \rightarrow(2 \pi)^{2} x$ as 
$x \rightarrow+\infty$ and $g(x) \rightarrow(3 \pi)^{2} x$ as $x \rightarrow-\infty$. The limiting equation (7) is now $z^{\prime \prime}+(2 \pi)^{2} z^{+}-(3 \pi)^{2} z^{-}=0$. Notice that the solution $z^{*}(t)$ with $z(0)=0$, $z^{\prime}(0)=1$ has exactly 2 zeros in $(0,1)$ and does not vanish at $t=1$. So does the solution $z_{*}(t)$ with $z(0)=0, z^{\prime}(0)=-1$. Therefore the problem is 2 nonresonant. The boundary value problem has at least $(7-2)+(7-2)+1$ solutions, the trivial one counted.

The following result relates to the case where the problem (2), (3) is under symmetric $k$-resonance.

Theorem 6.2. Let the condition (A3) hold and the problem (2), (3) be under symmetric k-resonance. Suppose also that the problem (2), (3) is under internal $n$-resonance.

Then there exist at least $2(n-k)-1$ solutions to the problem (2), (3), $\xi(t)$ counted.

Proofs of Theorems 6.1 and 6.2 are carried out by considering the behavior of the respective angular functions.

In a similar manner various combinations of resonances can be considered. Also, examples can be constructed showing that the estimation of the number of solutions are sharp.

Example. Consider the boundary value problem

$$
\begin{aligned}
& x^{\prime \prime}+x\left(1+x^{4} \sin \frac{1}{x}\right)=0, \\
& x(0)=0, \quad x(\pi)=0,
\end{aligned}
$$

in the interval $[0, \pi]$, where the right side of the equation is defined at $x=0$ by continuity. The equation of variations with respect to a trivial solution is

$$
y^{\prime \prime}+y=0
$$

and the problem (18) is under 1-internal resonance. Standard calculations show that the first zero function $t_{1}(\lambda)$ of solutions, defined by the initial data $x(0)=0$, $x^{\prime}(0)=\lambda$, oscillates near $t=\pi$ like $\sin \frac{1}{\lambda}$. Hence (18) has an infinite number of solutions.

\section{REFERENCES}

1. S. R. Bernfeld and V. Lakshmikantham, An introduction to nonlinear boundary value problems. Mathematics in Science and Engineering, Vol. 109. Academic Press, Inc. [A subsidiary of Harcourt Brace Jovanovich, Publishers], New York-London, 1974.

2. C. De Coster and P. Habets, Two-point boundary value problems: lower and upper solutions. Mathematics in Science and Engineering, 205. Elsevier B. V., Amsterdam, 2006.

3. S. FUČIK, Solvability of nonlinear equations and boundary value problems. Mathematics and its Applications, 4. D. Reidel Publishing Co., Dordrecht-Boston, Mass., 1980.

4. P. Habets, R. Manásevich, and F. Zanolin, A nonlinear boundary value problem with potential oscillating around the first eigenvalue. J. Differential Equations 117(1995), No. 2, 428-445. 
5. P. Habets, M. Ramos, and L. Sanchez, Jumping nonlinearities for Neumann BVPs with positive forcing. Nonlinear Anal. 20(1993), No. 5, 533-549.

6. D. C. Hart, A. C. Lazer, and P. J. McKenna, Multiple solutions of two-point boundary value problems with jumping nonlinearities. J. Differential Equations 59(1985), No. 2, 266-281.

7. D. C. Hart, A. C. Lazer, and P. J. McKenna, Multiplicity of solutions of nonlinear boundary value problems. SIAM J. Math. Anal. 17(1986), No. 6, 1332-1338.

8. C. FABRY and A. FONDA, Nonlinear equations at resonance and generalized eigenvalue problems. Nonlinear Anal. 18(1992), No. 5, 427-444.

9. C. Fabry, A. Fonda, and F. Munyamarere, Semilinear equations at resonance with nonsymmetric linear part. J. Math. Anal. Appl. 180(1993), No. 1, 189-206.

10. C. FABRY, Landesman-Lazer conditions for periodic boundary value problems with asymmetric nonlinearities. J. Differential Equations 116(1995), No. 2, 405-418.

11. I. T. Kiguradze, Some singular boundary value problems for ordinary differential equations. (Russian) Tbilisi University Press, Tbilisi, 1975.

12. Yu. A. Klokov and N. I. VAsilyev, Foundations of the theory of boundary value problems in ordinary differential equations. (Russian) Zinatne, Riga, 1978.

13. M. A. Krasnosel'skit, A. I. Perov, A. I. Povolockit, and P. P. Zabreiko, Plane vector fields. Translated by Scripta Technica, Ltd. Academic Press, New York-London, 1966.

14. E. M. LANDESman and A. C. Lazer, Nonlinear perturbations of linear elliptic boundary value problems at resonance. J. Math. Mech. 19(1969/1970), 609-623.

15. A. C. LAzer, Solvability of nonlinear equations and boundary value problems, by S. Fučik, Mathematics and its Applications, 4. D. Reidel Publishing Co., Dordrecht-Boston, Mass., 1980. In: Book Reviews, Bull. (N.S.) Amer. Math.Soc. 8(1983), No. 3, 483-489.

16. D. Lupo and M. Ramos, Some multiplicity results for two-point boundary value problems near resonance. Semin. Math., Inst. Math. Univ. Cathol. Louvain, No. 165, 1989.

17. R. MA and B. Thompson, Multiplicity results for second-order two-point boundary value problems with nonlinearities across several eigenvalues. Appl. Math. Lett. 18(2005), No. 5, 587-595.

18. J. Mawhin and W. Omana, Two-point boundary value problems for nonlinear perturbations of some singular linear differential equations at resonance. Comment. Math. Univ. Carolin. 30(1989), No. 3, 537-550.

19. M. Ramos, Remarks on resonance problems with unbounded perturbations. Differential Integral Equations 6(1993), No. 1, 215-223.

20. B. Ruf, Multiplicity and eigenvalue intersecting nonlinearities. Z. Angew. Math. Phys. 40(1989), No. 5, 774-779.

21. F. Sadyrbaev, Multiplicity of solutions for two-point boundary value problems with asymptotically asymmetric nonlinearities. Nonlinear Anal. 27(1996), No. 9, 999-1012.

22. R. SchaAf and K. Schmitt, A class of nonlinear Sturm-Liouville problems with infinitely many solutions. Trans. Amer. Math. Soc. 306(1988), No. 2, 853-859.

(Received 11.01.2007)

Author' address:

Institute of Mathematics and Computer Science, University of Latvia

Rainis blvd. 29, LV-1459 Riga, Latvia

E-mail: felix@cclu.lv 